\title{
Production of Substances by Medicago truncatula that Affect Bacterial Quorum Sensing
}

\author{
Mengsheng Gao, ${ }^{1}$ Max Teplitski, ${ }^{1}$ Jayne B. Robinson,, ${ }^{2}$ and Wolfgang D. Bauer ${ }^{1}$ \\ ${ }^{1}$ Department of Horticulture and Crop Science, 2021 Coffey Rd., Ohio State University, Columbus, OH 43210 U.S.A.; \\ ${ }^{2}$ Biology Department, University of Dayton, Dayton, OH 45460-0002 U.S.A.
}

Submitted 24 February 2003. Accepted 23 May 2003.

Earlier work showed that higher plants produce unidentified compounds that specifically stimulate or inhibit quorum sensing (QS) regulated responses in bacteria. The ability of plants to produce substances that affect QS regulation may provide plants with important tools to manipulate gene expression and behavior in the bacteria they encounter. In order to examine the kinds of QS active substances produced by the model legume $M$. truncatula, young seedlings and seedling exudates were systematically extracted with various organic solvents, and the extracts were fractionated by reverse phase $C_{18}$ high-performance liquid chromatography. $M$. truncatula appears to produce at least 15 to 20 separable substances capable of specifically stimulating or inhibiting responses in QS reporter bacteria, primarily substances that affect QS regulation dependent on $\mathbf{N}$-acyl homoserine lactone (AHL) signals. The secretion of AHL QS mimic activities by germinating seeds and seedlings was found to change substantially with developmental age. The secretion of some mimic activities may be dependent upon prior exposure of the plants to bacteria.

Quorum sensing (QS) in bacteria involves the regulation of genes and behaviors in response to changes in the local number and proximity of siblings and allows cooperation among members of a community (Miller and Bassler 2001; Whitehead et al. 2001). QS is dependent on the synthesis, exchange, and perception of small signal molecules between bacteria. These QS signals typically activate specific receptors that function as transcriptional regulators. In gram-negative bacteria, which are responsible for most plant and animal diseases, the most common QS signals are $N$-acyl homoserine lactones (AHLs) (Whitehead et al. 2001). Many bacterial plant pathogens and plant-associated bacterial species have been found to make AHLs (Cha et al. 1998; Pierson et al. 1999). Random mutagenesis revealed that over 250 different genes were affected by AHL-mediated QS in Pseudomonas aeruginosa, a pathogen of both humans and plants (Rahme et al. 2000; Whiteley et al. 1999). Recent transcriptome analyses indicate that 6 to $10 \%$ of the $P$. aeruginosa genome is affected by QS (Schuster et al 2003, Wagner et al 2003). Thus, AHL signaling is both common among relevant bacteria and can affect many aspects of their growth, survival, and interactions with eukaryotes.

Importantly, eukaryotic organisms are able to produce and secrete compounds that mimic the QS signals of bacteria and thus affect the behavior of associated bacteria (Bauer and Robinson 2002; Bauer and Teplitski 2001; Kjelleberg and

Corresponding author: W.D. Bauer; E-mail: bauer.7@ osu.edu.
Steinberg 2002). The halogenated furanones of Delisea pulchra, a marine red alga, were observed to share structural similarity with bacterial AHL and were shown to strongly and specifically inhibit QS-regulated behaviors in a variety of bacterial species (Givskov et al. 1996). The furanones were reported to interact with bacterial AHL receptors (Manefield et al. 1999) and to promote their rate of proteolytic degradation (Manefield et al. 2002) in contrast to the stabilizing effect of AHL signal binding (Zhu and Winans 2001). More recently, higher plants, such as pea, crown vetch, $M$. truncatula, rice, soybean, and tomato, were also shown to produce substances that appear to mimic the activities of AHL and have specific effects on QSregulated behaviors in bacteria (Daniels et al. 2002; Teplitski et al. 2000). In contrast to Delisea pulchra, higher plants secreted substances that specifically stimulated AHL-dependent QS responses, as well as substances that inhibited such responses. The chemical structures and mode of action of these AHL "signalmimic" compounds from plants have not been determined, although it appeared likely that most of the active compounds from pea are chemically different than bacterial AHLs and probably interact directly with AHL receptors (Teplitski et al. 2000).

In this report, we provide an initial characterization of the production and secretion of QS signal-mimic substances by the model legume $M$. truncatula. M. truncatula is being widely adopted as a system for molecular analysis of plant-microbe interactions, including the $\mathrm{N}$-fixing bacterial symbiont $\mathrm{Si}$ norhizobium meliloti, symbiotic mycorrhizae, and various bacterial and fungal pathogens (Cook 1999). We have used wellcharacterized QS reporter strains to detect a diversity of QS mimic activities in exudates and extracts of $M$. truncatula seedlings and to provide evidence for developmentally regulated secretion of some of the signal-mimics.

\section{RESULTS}

Microbial contamination of seedlings.

In addition to routine tests for microbial contaminants in the water used to grow and rinse the seedlings, seeds were surface-sterilized twice, as described below, and were germinated individually on $0.5 \%$ agar in $1-\mathrm{cm}$ microtiter plate wells. After 4 days, the young seedlings were rinsed, the liquid tested for contaminants, and the seedlings crushed in the wells to test for potential endophytic or tightly adhering contaminants. None of the 96 individual crushed seedlings or rinse liquids showed evidence of microbial contaminants. This provides further assurance the QS active compounds detected in this study by systematic extraction of seedlings and exudates (Fig. 1) were of plant origin, not from microbes growing in or on the seedlings. 
Detection of QS signal-mimics

in methanol extracts of seedling exudates.

As shown in Figure 2, several separable substances capable of selectively stimulating different QS reporters were detected in methanol extracts of lyophilized seedling exudate when tested with 15 to 30 "seedling equivalent" amounts of sample. Seedling equivalents were calculated based on the number of seedlings that contributed to the material injected on the column, the volume of a fraction taken for bioassay, and on the simplifying assumptions that the recovery of each compound for injection was $100 \%$ and that each compound eluted in only one fraction. The Pseudomonas putida CepR reporter (Table 1) was modestly stimulated by compounds in fraction 19 (Fig. 2), and responses to its cognate AHL were appreciably inhibited by substances in fraction 26 but not by substances in fractions 8 to 15 (data not shown). The E. coli LuxR reporter was strongly stimulated by compounds in fractions 24 to 25 and 47 to 51 (Fig. 2) and was strongly inhibited by compounds eluting in fractions 21 to 24 (Table 2). The $E$. coli LasR reporter was also stimulated by substances in fractions 24 to 25 and in later fractions (47 to 57). No activities were detected in any of the high-performance liquid chromatography (HPLC) fractions from solvent only or evaporated solvent control runs (discussed below), indicating that the active substances were plantderived and not present in the solvents nor solubilized from the column. The Vibrio harveyi BB170 reporter, which responds to the furanosyl borate diester AI-2 QS signal (Chen et al. 2002) and unknown substances in Salmonella culture filtrates, was modestly stimulated by substances in peaks eluting at fractions 32 and 39 (Table 2). The E. coli AhyR and Chromobacterium violaceum $\mathrm{CviR}$ reporters detected no stimulatory activities in any of the HPLC fractions of the methanol extract (data not shown). The responses of these two reporters to their cognate AHL were substantially inhibited by substances in fractions 8 to 15 (data not shown).

\section{SEEDLINGS}

$\Downarrow$

$\begin{array}{ll}\begin{array}{l}\text { Transfer } \Rightarrow \\ \text { seedlings } \\ \text { to EtOAc }\end{array} & \begin{array}{l}\text { HPLC } \\ \text { EtOAc } \\ \text { seedling } \\ \text { extract }\end{array} \\ \qquad & \\ \begin{array}{l}\text { Transfer } \\ \text { extracted } \\ \text { seedlings } \\ \text { to MeOH }\end{array} & \begin{array}{l}\text { MeOC } \\ \text { seedling } \\ \text { extract }\end{array}\end{array}$

$\Downarrow$

\begin{tabular}{|c|c|}
\hline $\begin{array}{l}\text { Transfer } \Rightarrow \\
\text { extracted } \\
\text { seedlings } \\
\text { to } 50 \% \\
\text { MeOH }\end{array}$ & $\begin{array}{l}\text { HPLC } \\
50 \% \\
\text { MeOH } \\
\text { seedling } \\
\text { extract }\end{array}$ \\
\hline
\end{tabular}

\section{SEEDLING RINSATE}

$\Downarrow$

$\begin{array}{ll}\begin{array}{l}\text { Extract } \\ \text { freeze dried } \\ \text { rinsate with }\end{array} & \begin{array}{l}\text { HPLC } \\ \text { EtOAc } \\ \text { EtoAc }\end{array} \\ \text { rinsate } \\ \text { extract }\end{array}$

$\Downarrow$

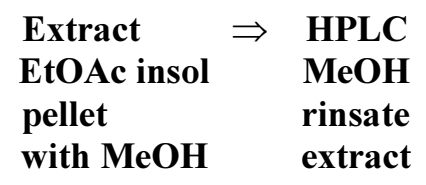

$\Downarrow$

$\begin{array}{lll}\text { Extract } & \Rightarrow & \text { HPLC } \\ \text { MeOH insol } & \mathbf{5 0 \%} \\ \text { pellet with } & \text { MeOH } \\ \mathbf{5 0 \% ~ M e O H} & \begin{array}{l}\text { rinsate } \\ \text { extract }\end{array}\end{array}$

Detection of QS mimics

in ethyl acetate and $50 \%$ methanol extracts of exudate.

LuxR stimulatory activities detected in ethyl acetate extracts of lyophilized exudate are shown in Figure 3A, while those detected with the LasR and LuxP reporters are indicated in Table 2. The LuxR stimulatory compounds in the ethyl acetate extracts were found to elute from the $\mathrm{C}_{18}$ column in essentially the same fractions as those recovered in the methanol extracts (Fig. 2, Table 2). Based on the higher level of activity seen in corresponding fractions from the methanol extract, it seems likely that the LuxR active compounds were partially extracted with ethyl acetate but were more soluble in methanol. Several other stimulatory and inhibitory activities sharing similar retention times also appeared to be present in extracts with two different solvents (Table 2). It remains to be established in each case whether a single active compound was extracted into both solvents or whether the different solvents extracted different compounds that coincidentally eluted with essentially the same retention time.

As illustrated in Figure 3B, the 50\% methanol extract of exudate appeared to contain several substances capable of inhibiting responses of the LuxR reporter to its cognate AHL. Compounds eluting in fractions 8 to 15 strongly inhibited the LasR, AhyR, CviR, and LuxP reporters, as well as the LuxR reporter, but did not inhibit the CepR reporter (data not shown), indicating that the inhibition is not likely the result of generally toxic substances. Reporter-specific inhibition of CviR responses by substances in the nonretained fractions of pea root exudate were observed in an earlier study (Teplitski et al. 2000). The substances in these early, minimally retained fractions inhibited LuxR luminescence to levels even below those of the reporter only controls. This low luminescence might be explained if the plant compounds enhanced the proteolytic degradation of the LuxR protein below the levels normally available from the multicopy plasmid, similar to the enhanced degradation of LuxR induced by the Delisea furanone mimics (Manefield et al. 2002). Figure 3B shows several other

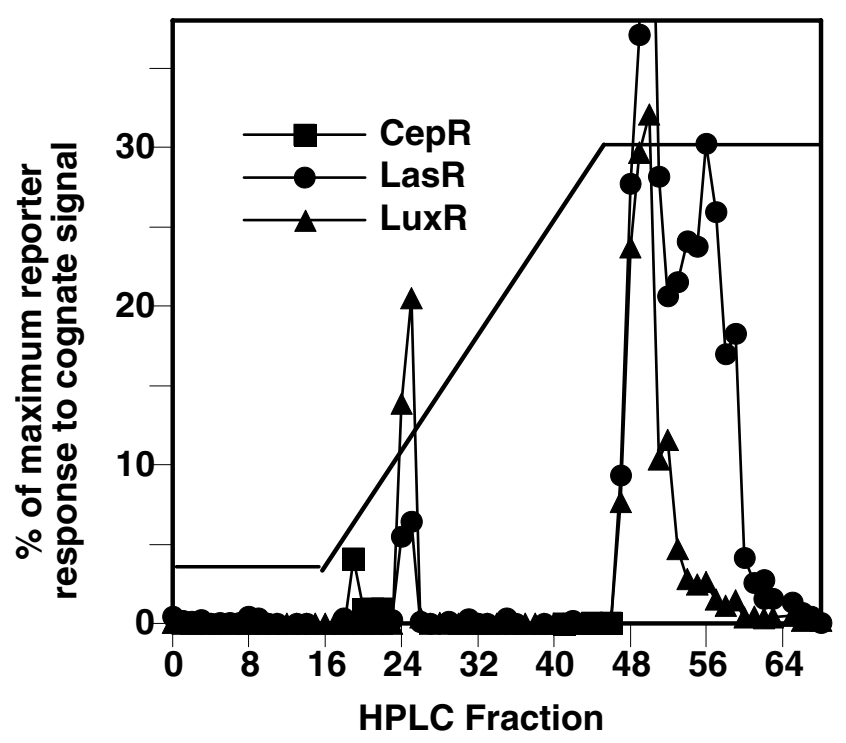

Fig. 2. Fractionation of quorum sensing (QS) signal-mimic activities in methanol extacts of Medicago truncatula seedling exudates. Fractions from a $\mathrm{C}_{18}$ reverse phase high-performance liquid chromatography column were collected, and samples equivalent to exudate from about 30 seedlings were assayed for activity. Responses to the plant compounds are reported as a percentage of maximum response of each reporter $(\mathrm{CepR}=$ square LasR $=$ circle $;$ LuxR $=$ triangle $)$ to a saturating concentration of its cognate QS signal. The solid line indicates the acetonitrile concentration (10 to $100 \%$ ) present in the collected fractions. Results shown are from a single experiment representative of three independent experiments.

Fig. 1. Outline of extraction, fractionation, and detection of quorum sensing active compounds from Medicago truncatula. 
peaks with moderate LuxR inhibitory activity in fractions eluting between 16 and $66 \mathrm{~min}$. These later fractions contained only one peak of LasR inhibitory activity (Table 2) and no AhyR or CepR inhibitory activities (data not shown). Thus, these peaks could correspond to separate LuxR-specific mimics or inhibitors. However, before accepting these four or five peaks of moderate LuxR inhibitory activity as separate putative mimic substances, it will be necessary to individually refractionate these activity peaks to establish that they are not artifacts resulting from column overloading or trailing of the very large ( $>80 \%$ total $\mathrm{A}_{220}$ material) peak in fractions 8 to 15 .

It may be helpful to note that bioassays of crude seedling exudate preparations or extracts have not provided useful measures of net activity.

\section{QS mimics in extracts of seedlings.}

After collection of the exudates, the 7-day-old seedlings that generated the exudates were sequentially extracted with ethyl acetate, methanol, and 1:1 methanol/water to determine whether M. truncatula accumulated QS mimics inside the plant tissues and to learn whether the accumulated mimics corresponded to the secreted mimics. As shown in Table 2, seedling extracts contained several activities that eluted in fractions similar to activities in the exudate extracts. For example, the methanol extracts of both seedlings and exudates contained a LasR stimulatory activity that peaked at about fraction 25 , and the ethyl acetate extracts of both seedlings and exudates contained a LuxP stimulatory activity peak that eluted at fraction 32 (Table 2). However, many activities in the seedling extracts had no obvious counterparts in the exudate extracts and vice versa. For example, the ethyl acetate and methanol extracts from exudates contained LuxR stimulatory activities eluting in peaks at fractions 25,51 , and 54 that were not matched by anything in extracts from seedlings (Table 2).

\section{Further fractionation of LasR stimulatory mimics.}

In order to further purify the plant compounds responsible for the strong LasR stimulatory activities eluting at high acetonitrile concentrations (Fig. 2) for chemical identification and to learn more about the number of active compounds actually present in these fractions, the material present in fractions 44 to 61 was pooled and subjected to isocratic fractionation at $80 \%$ acetonitrile. As shown in Figure 4, the largest, partially resolved LasR activity peaks seen in Figure 2 were almost completely separated after such refractionation. However, when the amount of material assayed was increased to the equivalent of approximately 70 seedlings, fractions showing little activity at 25 to 50 seedling equivalent levels were found to strongly stimulate the reporter, indicating the presence of additional LasR stimulatory mimic compounds in these fractions.

\section{Changes in secretion of QS signal-mimic substances during seed germination and seedling development.}

When individual seeds were inoculated with the AhyR reporter at the time of transfer from $4^{\circ} \mathrm{C}$ to room temperature and were incubated for $4 \mathrm{~h}$, substances present on or near the seed surface inhibited the luminescence response of the reporter to added $\mathrm{C}_{4}$-HL by about twofold. This net inhibitory activity increased to about 10 -fold when the seeds were inoculated at $6 \mathrm{~h}$ post-transfer. Seedlings inoculated at $24 \mathrm{~h}$ after the initiation of germination again gave a net inhibitory response of about twofold, but substances on the surface of seedlings inoculated with the AhyR reporter $48 \mathrm{~h}$ after the onset of germination stimulated the reporter about 15-fold. These changes in net QS responses are averages based on measurements of 20 individual seeds or seedlings in repeated experiments. Responses to individual seeds and seedlings varied over a two- to fourfold range.

The LasR stimulatory substances secreted by seeds and seedlings also changed substantially during various stages of development. As shown in Figure 5, exudates collected from germinating seed prior to emergence of the radicle $(0$ to $6 \mathrm{~h}$ ) contained low levels of a few LasR stimulatory compounds eluting primarily in fraction 20 , fractions 48 to 58 , and fractions 60 to 66 . When exudate was collected from the same seedlings during the interval from 6 to $24 \mathrm{~h}$, the LasR mimic activities seen in the 0 to $6 \mathrm{~h}$ exudate were no longer detected. Instead, the seedlings secreted mimic activities appearing in fractions 38 to 44 . These LasR mimic activities were not detectable in the exudate secreted during the interval from 24 to $48 \mathrm{~h}$. This exudate instead contained LasR stimulatory compounds that eluted in fractions 23 to 24 and 48 to 55. There appears to be some limited overlap of the LasR stimulatory compounds detected in the exudate after 7 days and those detected earlier.

\section{DISCUSSION}

Factors affecting the recovery, detection, and quantification of QS signal/signal-mimic compounds produced by $M$. truncatula.

Reliable detection and quantification of QS active compounds from the plant is made difficult in at least some cases by nonlinear responses of the reporters. As evident from the results in Figure 4, doubling the amount of the sample assayed from 25 seedling equivalents to 50 seedling equivalents increased the response of the LasR reporter to the compounds in peak fractions 22 and 24 by almost 10 -fold. Increasing the amount of sample further from 50 to 70 seedling equivalents approximately doubled the responses to compounds in these peak fractions. In addition, the increase from 50 to 70 seedling equivalents gave five- to 10 -fold higher responses in fractions 18 to 21,23 , and 25 to 30 , suggesting that these fractions may contain additional LasR stimulatory compounds. We speculate that these compounds are detected only at higher sample concentrations because they have lower affinities for the LasR receptor. If this is correct, then the number of different QS signal-mimic compounds actually produced by the plant may be several times larger than the estimate of 15 to 20 provided by the results in Table 2. It also makes the amount of sample taken for testing both crucial and somewhat arbitrary.

Table 1. Quorum-sensing reporter strains

\begin{tabular}{|c|c|c|c|}
\hline Bacterium & Receptor & Cognate Signal & Source \\
\hline Chromobacterium violaceum $\mathrm{CV} 026$ & CviR & $\mathrm{C}_{6}-\mathrm{HL}$ & McClean et al. 1997 \\
\hline Pseudomonas putida pAS-C8 & CepR & $\mathrm{C}_{8}-\mathrm{HL}$ & Steidle et al. 2001 \\
\hline Vibrio harveyi $\mathrm{BB} 170$ & LuxP & AI-2 furanosyl borate ester & Bassler et al. 1993 \\
\hline Escherichia coli JM109 & & & Winson et al. 1998 \\
\hline $\mathrm{p}(\mathrm{SB} 401)$ & LuxR (Vibrio) & 3-oxo- $\mathrm{C}_{6}$-HSL & \\
\hline $\mathrm{p}(\mathrm{SB} 536)$ & AhyR (Aeromonas) & $\mathrm{C}_{4}-\mathrm{HSL}$ & \\
\hline $\mathrm{p}(\mathrm{SB} 1075)$ & LasR ( $P$. aeruginosa) & 3-oxo- $\mathrm{C}_{12}$-HSL & \\
\hline
\end{tabular}


The reporters used in the present studies gave reasonably consistent results with 15 to 30 seedling equivalent amounts of material per assay well. It is difficult to know how the concentration of a given QS active compound in the assay wells at 15 to 30 seedling equivalents compares with the concentration of the same compound in the thin film of liquid on a root growing in soil. It is possible that they are fairly similar. When the LasR, AhyR, and LuxR reporters were inoculated onto the surface of live, aseptically grown $M$. truncatula seedlings and the excess liquid blotted, compounds secreted by the seedlings induced the reporter cells to luminesce at 1 to $15 \%$ of the saturating cognate AHL controls (Teplitski et al. 2000). Thus, on individual seedlings, the surface concentration of the mixture of secreted stimulatory mimics was high enough to activate the reporters, despite the effects of any inhibitory or toxic compounds. The volume of liquid adhering to the seedling surfaces in these experiments was perhaps one-fourth to one-eighth the volume of liquid in the assay wells $(80 \mu \mathrm{l})$ in the present studies. The simplifying assumptions made in calculating seedling equivalents (that $100 \%$ of each compound secreted by an individual seedling is perfectly recovered and ends up in a single HPLC fraction) probably overestimate the amount of substance in assay wells by a factor of two to four. Combined with a factor of four to eight in terms of liquid volume, it appears that the compounds detected by the reporters at 15 to 30 seedling equivalents of material in vitro may be present on the plant surface at concentrations high enough to be biologically relevant. Once the chemical identities of the QS active compounds are known, it may be possible to measure their surface concentration, as was done for the halogenated furanone mimics of Delisea pulchra (Dworjanyn et al. 1999).

None of the solvents tested, including tetrahydrofuran (data not shown) were able to readily solubilize all of the QS active compounds. While most of the active compounds reported here were retained at least slightly on the $\mathrm{C}_{18}$ reverse-phase column, the strong inhibitory activities in the $50 \%$ methanol extracts of seedling exudates were not appreciably retained by the column (Fig. 3A, Table 2). Other forms of chromatography will be required to determine the number and nature of QS active compounds among the compounds not retained by the $\mathrm{C}_{18}$ column. Most of the signal-mimic activities detected with the LasR and LuxR reporters in $M$. truncatula exudates have proven reasonably stable during isolation, fractionation, and storage at $-20^{\circ} \mathrm{C}$ for over a month, although their activity does appear to decline slowly with storage. None of the activity peaks retained by the column and eluting at acetonitrile concentrations $>15 \%$ were correlated with an absorbance peak at $220 \mathrm{~nm}$ (data not shown).
Specificity and mechanism of responses elicited by QS active compounds from $M$. truncatula.

The stimulatory and inhibitory substances detected with the QS reporters in this study were found to affect only one or several of the reporters but not all six of them. This selectivity suggests that the active compounds are probably affecting QS regulation in the reporter bacteria in some specific way. With the LasR, LuxR, AhyR, and CepR reporters, the plant substances that stimulated luminescence or green fluorescent protein (GFP) fluorescence in the reporter almost certainly did so in a receptor-mediated fashion similar to activation by the cognate AHL, since there is no plausible alternative for these constructs. It is unlikely that any of the observed stimulatory activities could be explained on the basis of enhanced growth of the reporter bacteria by "nutritious" plant compounds in the fractions. The reporters were all suspended in rich Luria Bertani (LB) medium at rather low cell density for the bioassays. Thus, the reporter bacteria had all the nutrients they needed for optimal, sustained growth for many hours. In order to account for the observed stimulatory effects, any plant compound would have to enhance growth (and thus background reporter luminescence or fluorescence) to levels two- to 50-fold over their level of growth in LB medium. In addition, the three E. coli reporters were not all stimulated equally by substances in the HPLC fractions, as they should be in response to any "growth enhancing" plant substance, since they are the same bacterium with the same plasmid, differing only in the DNA sequence for the specific AHL receptor gene and synthase promotor.

Compounds that inhibited the responses of the reporters to partially inducing levels of the cognate QS signals could be simply toxic, or they could nonspecifically inhibit luminescence, fluorescence, or other reporter functions, or they could interact directly with the receptor protein as authentic QS mimics. In those cases where a plant compound inhibited the responses to a cognate AHL in just one but not all of the E. coli reporters, the compound can reasonably be assumed to have specific, receptor-mediated effects on QS regulation.

The specificity and mechanism of action of the plant compounds that stimulated the $V$. harveyi LuxP AI-2 reporter are quite uncertain. In a previous report (Mathesius et al. 2003), $M$. truncatula was shown to secrete compounds that inhibited the responses of the LuxP reporter to the "AI-2" signal in $S$. enterica culture filtrates. Many bacterial species contain homologs of LuxS, the $V$. harveyi AI-2 synthase, and can make compounds that stimulate the LuxP reporter (Miller and Bassler 2001). However, it is not yet clear whether these other species actually synthesize AI-2 and have specific receptors for

Table 2. Quorum sensing (QS) signal-mimic activities detected in seedlings and exudates of M. truncatula

\begin{tabular}{|c|c|c|c|c|c|}
\hline \multirow[b]{3}{*}{ QS reporter activity } & \multicolumn{5}{|c|}{ QS reporter responses to $M$. truncatula compounds ${ }^{\mathrm{a}}$} \\
\hline & \multicolumn{2}{|c|}{ LuxR } & \multicolumn{2}{|c|}{ LasR } & \multirow{2}{*}{$\begin{array}{c}\text { LuxP } \\
\text { Stimulatory }\end{array}$} \\
\hline & Stimulatory & Inhibitory & Stimulatory & Inhibitory & \\
\hline \multicolumn{6}{|l|}{ In seedling extracts } \\
\hline EtOAc & $\mathrm{ND}^{\mathrm{b}}$ & ND & Fr. $49^{\mathrm{c}} \mathrm{S}$ & ND & Fr $32 \mathrm{~S}$, Fr $34 \mathrm{~S}$ \\
\hline $100 \% \mathrm{MeOH}$ & Fr 36W & Fr $29 \mathrm{~S}$, Fr $61 \mathrm{~S}$ & Fr $25 \mathrm{~W}$, Fr $48 \mathrm{~S}$ & Fr $17 \mathrm{~W}$ & ND \\
\hline $50 \% \mathrm{MeOH}$ & Fr 36S & Fr10 S & Fr $40 \mathrm{~W}$, Fr $47 \mathrm{~W}$ & Fr $10 \mathrm{~S}$, Fr $31 \mathrm{~S}$ & Fr $25 \mathrm{M}$, Fr $28 \mathrm{~W}$ \\
\hline \multicolumn{6}{|l|}{ In exudate extracts } \\
\hline EtOAc & Fr $25 \mathrm{~W}$, Fr $51 \mathrm{~W}$, Fr $54 \mathrm{~W}$ & ND & Fr $54 \mathrm{M}$ & ND & Fr $32 \mathrm{~W}$ \\
\hline $100 \% \mathrm{MeOH}$ & Fr $25 \mathrm{M}$, Fr $51 \mathrm{~S}$, Fr $53 \mathrm{M}$ & Fr $21 \mathrm{~S}$ & Fr $25 \mathrm{~W}, 515,575$ & ND & Fr $32 \mathrm{~W}, 39 \mathrm{~W}$ \\
\hline $50 \% \mathrm{MeOH}$ & Fr $39 \mathrm{~S}$ & Fr $10 \mathrm{~S}$ & Fr $42 \mathrm{M}$, Fr $48 \mathrm{~W}$ & Fr10 S & ND \\
\hline
\end{tabular}


it that play a role in QS regulation (Whitehead et al. 2001; Winzer et al. 2002). If future studies show that such bacteria do use AI-2 or analogs of it for QS, then the secretion of "mimics" that activate or inhibit LuxP could be important to higher plants in their interactions with such bacteria.

Very few of the QS-active compounds detected in this study were extracted by ethyl acetate, and several of those extracted by ethyl acetate appeared to be more soluble in methanol (Table 2). Bacterial AHLs, on the other hand, are preferentially extracted with ethyl acetate. Thus, it appears that the QS-active plant compounds that preferentially extracted into methanol or $50 \%$ methanol are chemically different than bacterial AHLs. Preliminary attempts to detect AHLs in samples of purified LasR stimulatory mimics, using an electrospray ionization MS/MS method for AHL identification (Marketon et al 2002) were negative (A. Eberhard, M. Gronquist, M. Teplitski, and



Fig. 3. Quorum sensing signal-mimic activities detected with LuxR in ethyl acetate and 50\% methanol extracts of Medicago truncatula seedling exudates. Samples equivalent to about 30 seedlings were assayed with the LuxR reporter to detect $\mathbf{A}$, stimulatory activities in the ethyl acetate extract or $\mathbf{B}$, inhibitory activities in the $50 \%$ methanol extract in fractions from $\mathrm{C}_{18}$ reverse phase high-performance liquid chromatography. Responses to stimulatory plant compounds are reported as a percentage of maximum response of the LuxR reporter to a saturating concentration of 3-oxo- $\mathrm{C}_{6}-\mathrm{HL}$. Responses to inhibitory compounds are reported as the percentage inhibition of LuxR responses to $4.7 \mathrm{nM} 3-$ oxo- $_{6}$-HL. The solid line indicates the acetonitrile concentration (10 to $100 \%$ ) present in the collected fractions. Results shown are from a single experiment representative of three independent experiments.
W. D. Bauer, unpublished data). Based on these considerations, it seems likely that most or all of the active compounds detected in this study are not AHLs but authentic, plant-derived AHL signal-mimic compounds capable of specifically affecting QS regulation in associated bacteria.

\section{Production and secretion}

of QS active compounds by M. truncatula.

Table 2 provides evidence that $M$. truncatula produces more than a dozen separable compounds that stimulate or inhibit QS responses in the LasR, LuxR, or LuxP reporters. However, the purity and retention time of each individual activity peak needs to be established by refractionation and assay at different concentrations, as seen in Figure 4, before we can be confident that each activity is distinct. The results in Figures 4 and 5 indicate that $M$. truncatula may produce additional QS active compounds not shown in Table 2 . Tests with additional reporter strains of differing signal recognition specificities are also likely to reveal more QS signal-mimic substances. The red alga Delisea pulchra has been reported to synthesize about 30 different halogenated furanones at various levels of abundance (Kjelleberg and Steinberg 2002), so the production of a substantial diversity of QS mimics by a higher plant is not surprising. It appears that all of the QS active compounds produced by Delisea pulchra belong to a single chemical class of compounds. It remains to be seen whether the M. truncatula mimics likewise belong to a single class of compounds.

In considering the diversity of QS active compounds that $M$. truncatula is capable of making, it is important to recognize that the plants examined in this study were grown in the absence of microbes. It is well known that plants alter the kinds and levels of secondary metabolites they produce in response to the presence of microbes. Thus, the presence of microbes may stimulate the plant to synthesize or secrete yet additional QS active com-



Fig. 4. LasR responses to different amounts of stimulatory substances after refractionation. The LasR stimulatory substances in fractions 44 to 61 (Fig. 2) were pooled and refractionated on the same $\mathrm{C}_{18}$ column by isocratic elution with $4: 1$ acetonitrile/water. Samples equivalent to exudates from about 25 seedlings (triangle), 50 seedlings (circle), or 70 seedlings (square) were assayed with the LasR reporter. Responses to stimulatory plant compounds are reported as a percentage of maximum response of the LasR reporter to a saturating concentration of 3-oxo- $\mathrm{C}_{12}$ HL. Results shown are from a single experiment representative of two independent experiments. 
pounds or different amounts of the same compounds. In this regard, exposure of $M$. truncatula seedlings to nanomolar levels of AHLs stimulated the plant to secrete different amounts and perhaps different kinds of LasR stimulatory and LuxP inhibitory compounds (Mathesius et al. 2003). In addition, we note that the AhyR reporter bacteria (E. coli) inoculated onto $M$. truncatula seedlings detected (and perhaps induced) the secretion of compounds capable of stimulating that reporter (Teplitski et al. 2000), even though no AhyR stimulatory mimics were detected after extraction of aseptically grown $M$. truncatula seedlings or exudates. The induced synthesis and secretion of QS active compounds by plants in response to contact with microbes may prove to be an important aspect of many plant-bacterial interactions.

Figure 5 indicates that $M$. truncatula seed and seedlings secreted different LasR stimulatory mimics or substantially different amounts of the same mimics at different stages of development. Since many of the LasR active compounds in the 0 to $24 \mathrm{~h}$ exudates from seed and young seedlings subsequently fell below the limits of detection, it appears that the plant stops either making or secreting them and that the initially secreted mimics are degraded or inactivated by unknown mechanisms during the next 7 days. The biological significance of these changes in secreted QS active compounds for interactions with naturally encountered bacteria remains to be investigated.

Comparison of the QS signal-mimic activities inside the plant with those recovered in the exudate (Table 2) makes it clear that, with few exceptions, the plant did not accumulate very much of the mimic compounds that it secreted and did not secrete very much of the QS active compounds that it accumulated. This suggests that the regulation of signal-mimic secretion may be a determining factor in how and when the plant deploys specific mimics to disrupt QS regulation in the bacteria it encounters.

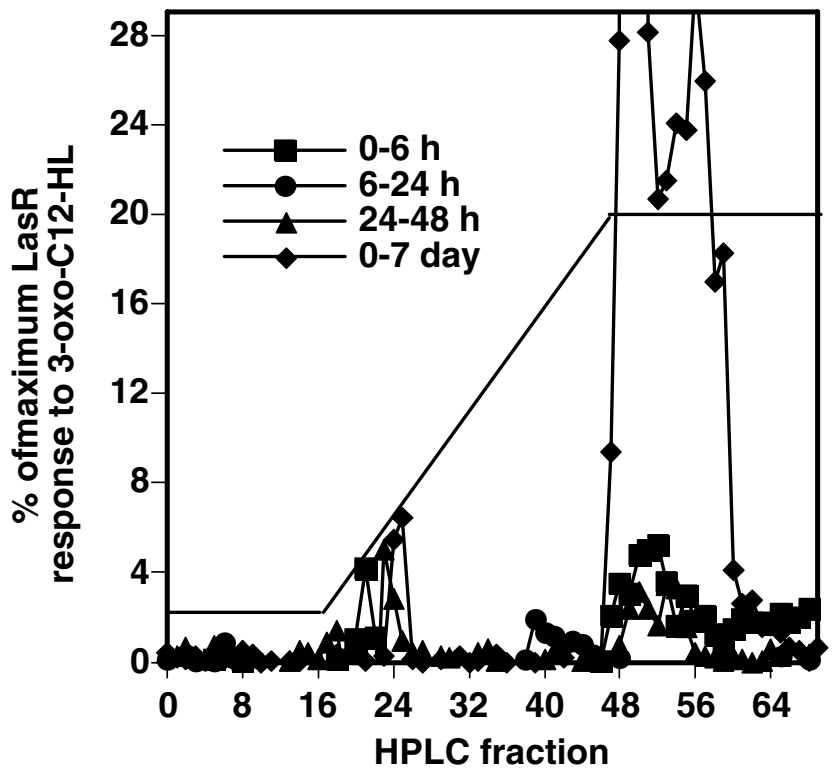

Fig. 5. Effect of seedling development on secretion of LasR stimulatory signal-mimic activities. Surface-sterilized seeds were placed in a shallow layer of water and the compounds secreted during the interval from 0 to 6 $\mathrm{h}$ (square), from 6 to $24 \mathrm{~h}$ (circle), and from 24 to $48 \mathrm{~h}$ (triangle) were collected, freeze dried, extracted, and separated by reverse phase highperformance liquid chromatography, and samples equivalent to exudate from about 30 seeds or seedlings were assayed. Responses to the plant compounds are reported as a percentage of the maximum response of the LasR reporter to a saturating concentration of 3-oxo- $\mathrm{Cl}_{2}-\mathrm{HL}$. For comparison, the activities present in exudate collected from 7-day-old seedlings are also shown (diamond). The concentration of acetonitrile (10 to $100 \%$ ) present in the fractions is indicated by a solid line. Results shown are from a single experiment representative of three independent experiments.

\section{MATERIALS AND METHODS}

Surface sterilization and growth of plants.

Seeds of M. truncatula A17 (cv. Jemalong) were soaked in 95\% ethanol containing one drop of Tween 20 per $30 \mathrm{ml}$ for an hour, followed by surface sterilization with $0.1 \% \mathrm{HgCl}_{2}$ for 15 min. Surface-sterilized seeds were rinsed repeatedly with small volumes of sterile water and then with several large volumes over a 4-h period. Sterilized and imbibed seeds were kept for approximately $16 \mathrm{~h}$ at $4{ }^{\circ} \mathrm{C}$ to synchronize and enhance germination. After cold treatment, seeds were treated with $30 \%$ commercial bleach for $30 \mathrm{~s}$ and then rinsed repeatedly with sterile water. Batches of 150 to 200 imbibed, surface-sterilized seeds were grown aseptically in a pool of approximately $20 \mathrm{ml}$ water in $22 \times 30 \mathrm{~cm}$ Pyrex trays previously washed with $10 \% \mathrm{KOH}$ and 5\% HCL to remove the hydrophilic coating and anionic groups from the glass. The tray tops were covered with plastic film and $\mathrm{Al}$ foil and were incubated in an incubator with fluorescent lights at $24^{\circ} \mathrm{C}$. After 6 days, 200- $\mu$ l samples from each tray were transferred to LB agar plates to test for the presence of microbial contaminants. Exudates and seedlings from trays showing the growth of microbes on the test plates during the next four days were discarded. Typically, trays planted with 150 to 200 seeds gave germination rates of 70 to $80 \%$, and typically, about $10 \%$ of the trays developed microbial contamination. Without the second surface-sterilization step, 30 to $80 \%$ of the trays were contaminated (almost always by bacteria), making collection of uncontaminated exudate and seedlings cumbersome or problematic.

\section{Preparation of seedling exudates and extracts.}

As outlined in Figure 1, M. truncatula seedlings and seedling exudates were sequentially extracted with ethyl acetate, then methanol, and then $50 \%$ methanol/water, with samples of each extract fractionated by reverse phase HPLC prior to bioassay for signal-mimic activities. After 7 days, seedling exudate was collected by adding $70 \mathrm{ml}$ of sterile water to each tray and shaking gently for $30 \mathrm{~min}$. The exudate solution was decanted, centrifuged, was filtered through a glass fiber disk, was frozen, and lyophilized. The seedlings were then transferred to a beaker containing $30 \mathrm{ml}$ of ethyl acetate per 400 seedlings and were extracted for $30 \mathrm{~min}$ with gentle shaking at room temperature. The seedlings were subjected to a second such extraction in ethyl acetate, and then were transferred to methanol. After extraction twice in methanol, the seedlings were extracted twice in 1:1 methanol and water. The two extracts for each solvent were combined and were taken to dryness by rotary evaporation at room temperature. Similar volumes of pure solvent were taken to dryness for fractionation and bioassay as controls.

\section{Reverse phase $\mathrm{C}_{18}$ HPLC fractionation of seedling exudates and extracts.}

Lyophilized exudate was extracted with $3 \mathrm{ml}$ of ethyl acetate per 400 seedlings for 30 min with vortexing. Dryness of the lyophilized sample and extraction volume had significant effects on the extraction efficiencies. The suspension was centrifuged in microfuge tubes at high speed to remove insoluble material. The supernatant was saved at $-20^{\circ} \mathrm{C}$ for later evaporation, solubilization in $3 \mathrm{ml}$ of acetonitrile, and HPLC fractionation. The pellet was suspended in $3 \mathrm{ml}$ of methanol and was extracted for $30 \mathrm{~min}$ with vortexing at room temperature. After centrifugation, $2 \mathrm{ml}$ of the supernatant was removed for HPLC fractionation, and $1 \mathrm{ml}$ of water was added to the remaining methanol and pellet. After resuspension, extraction for $30 \mathrm{~min}$, and centrifugation, the $2 \mathrm{ml}$ of $50 \%$ methanol supernatant was removed for HPLC. In some cases, the lyophilized exudate was extracted directly with $3 \mathrm{ml}$ of methanol per 400 seedlings 
with no prior extraction with ethyl acetate. Two-ml samples of the ethyl acetate, methanol, and 50\% methanol extracts from 200 to 600 seedlings were injected onto a semipreparative $C_{18}$ column (Whatman Partisil 10 ODS-3) fitted with a guard column and equilibrated with $90 \%$ water and $10 \%$ acetonitrile. The column was eluted for 10 min with $90 \%$ water and $10 \%$ acetonitrile at $0.5 \mathrm{ml} / \mathrm{min}$, then $5 \mathrm{~min}$ at $1 \mathrm{ml} / \mathrm{min}$, then $30 \mathrm{~min}$ with a linear water and acetonitrile gradient at a flow rate of 1 $\mathrm{ml} / \mathrm{min}$ to $100 \%$ acetonitrile and then was maintained at $100 \%$ acetonitrile for $20 \mathrm{~min}$. Absorbance of the eluate was monitored at $220 \mathrm{~nm}$. Fractions were collected every minute and bioassayed with the reporters, as described below. Activity peaks were reproducible within \pm 1 min between duplicate runs. Fractions from solvent-only injections were assayed for active contaminants from the solvent and column. None were detected. Similarly, no activities were found in fractions from an injection of the substances present in $100 \mathrm{ml}$ of the pure ethyl acetate used for seedling extraction following evaporation of the solvent, for solubilization in $2 \mathrm{ml}$ acetonitrile, and for HPLC fractionation.

HPLC fractionation of the seedling extracts was performed in a similar manner. The dried residue from each extraction was resuspended and dissolved as well as possible at room temperature in $3 \mathrm{ml}$ of acetonitrile for the ethyl acetate extracts, $3 \mathrm{ml}$ of methanol, or $50 \%$ methanol and then centrifuged to remove any insoluble material. Two-ml samples of the supernatants, containing material from approximately 400 seedlings, were injected onto the semipreparative $\mathrm{C}_{18}$ column eluted with a linear water and acetonitrile gradient as described above. Fractions were stored at $-20^{\circ} \mathrm{C}$.

\section{Bioassay with QS reporters.}

Aliquots of each fraction equal to 15 to 30 seedling equivalents were transferred to wells in 96-well black microtiter plates. The plates were placed in a laminar flow hood for 6 to $12 \mathrm{~h}$ to evaporate the solvent.

The reporters used to detect substances affecting QS are listed in Table 1. To obtain sensitive and reproducible responses, some care was needed in preparing the reporters for bioassay. The three $E$. coli reporters were taken from glycerol stocks, were grown as shake cultures overnight at $37^{\circ} \mathrm{C}$ in $5 \mathrm{ml}$ of LB medium with the appropriate antibiotics, then were subcultured twice in rich LB medium, each time with a 10 -fold dilution. The second subcultures were allowed to grow to an optical density at $600 \mathrm{~nm}$ of 0.3 to 0.4 . These log phase cultures were then centrifuged and resuspended in fresh LB medium without antibiotic to give a 10 - to 20 -fold dilution. Assays with the E. coli LasR, LuxR, and AhyR reporters involved adding $80 \mu \mathrm{l}$ of the reporter suspension to each microtiter plate well containing a dried HPLC fraction or sample, incubating the plate at $37^{\circ} \mathrm{C}$ for 3 to $6 \mathrm{~h}$, and then measuring luminescence for $0.1 \mathrm{~s}$ per well in a Wallace Victor2 multimode plate reader (Perkin-Elmer, Gaithersburg, MD, U.S.A.). In assays for both stimulatory and inhibitory activities, wells containing only the reporter served as the negative control. In assays for stimulatory activities, the positive control wells contained sufficient levels of the cognate AHL to give a maximal response for that reporter. For detection of inhibitory activities, the positive controls consisted of wells with an amount of the cognate AHL that partially induced the reporter (to approximately 20 to $40 \%$ of the maximal luminescence response). For the LuxRI'::luxCDABE reporter, the full and partial AHL response control wells contained approximately $100 \mathrm{nM}$ and $5 \mathrm{nM}$, respectively, 3-oxo- $\mathrm{C}_{6}$-HL (Quorum Science, Coraville, IA, U.S.A.). The maximal and partial response controls contained 150 and $5 \mathrm{nM}$ 3-oxo- $\mathrm{C}_{12}$-HSL (Quorum Science) for the LasRI'::luxCDABE reporter and approximately $3 \mu \mathrm{M}$ and $4 \mathrm{nM}$
$\mathrm{C}_{4}$-HSL (Teplitski et al. 2000) for the AhyRI'::luxCDABE reporter. Typically, the maximal AHL response controls for the LuxR, LasR, and AhyR E. coli reporters gave luminescence responses 1,000-fold, 60-fold, and 100-fold, respectively, over the background luminescence levels in the reporter-only controls. Responses to saturating concentrations of the cognate signal normally reached a maximum within about 5 to $7 \mathrm{~h}$ and declined at longer times. The time dependence of the responses is quite complex, particularly in assays for inhibitory activities. The reporter background response and the reporter responses to the plant compound, to the partially inducing level of cognate AHL, and to the fully inducing level of the cognate AHL all follow different rates of increase to maximum. Thus, it is important to assay relative responses to the plant compound at a time when the reporter response to the saturating level of cognate AHL is reaching its maximum, not much earlier and not much later. The reporters usually responded faster to their cognate QS signal than to the plant compounds. Plant compounds that elicited significant responses after $7 \mathrm{~h}$ but not earlier would have escaped detection in our assays. Responses to duplicate samples were usually reproducible to within about $5 \%$.

The $V$. harveyi BB170 reporter strain, a luxQ mutant that cannot respond to its 3-OH- $\mathrm{C}_{4}-\mathrm{HL}$ QS signal but luminesces in response to its AI2 QS signal in a LuxP-mediated manner (Bassler et al. 1993) was cultured from a glycerol stock overnight in Autoinducer Bioassay liquid medium, was resuspended in fresh medium, and was diluted 5,000-fold, $80 \mu \mathrm{l}$ of the suspension per well was added, and luminescence was assayed between 3 and $5 \mathrm{~h}$ later (Surette and Bassler 1998). Culture supernatant from Salmonella enterica serovar typhimurium 14028, cultured as described (Joyce et al. 2000), was used for full and partial induction. Addition of $10 \mu \mathrm{l}$ of the supernatant per well typically gave luminescence responses 100 - to 500-fold higher than the reporter-only control. Background luminescence in the reporter-only controls reached high levels quite quickly, so that repeated readings at approximately 10 -min intervals was often useful. The $P$. putida $\mathrm{CepR}$ reporter (Steidle et al. 2001) was used as described (Steidle et al. 2001) and as above for the E. coli reporters, except that the final suspension was diluted $4 \times$ rather than $10 \times$. GFP-based fluorescence was measured with the Victor2 reader, using appropriate filters after 4 to $6 \mathrm{~h}$. The $C$. violaceum CV026 CviR reporter was used as previously described (Teplitski et al. 2000). Concentrations of antibiotics used were at $150 \mu \mathrm{g}$ of ampicillin, $20 \mu \mathrm{g}$ of tetracycline, $50 \mu \mathrm{g}$ of kanamycin, and 20 $\mu \mathrm{g}$ of gentamicin per $\mathrm{ml}$.

\section{ACKNOWLEDGMENTS}

We thank J. Metzger for use of his equipment and generous advice. Work presented in this paper was supported by a United State Department of Agriculture grant 2002-3531911559 and an Ohio Plant Biotechnology Consortium grant to W. D. Bauer and J. B. Robinson and a Presidential Fellowship, Ohio Agricultural Research and Development Center Research Enhancement Grant and Ohio State University Extension Sustainable Agriculture grant to M. Teplitski. Partial support for salary and supplies was also provided to W. D. Bauer by the Ohio Agricultural Research and Development Center.

\section{LITERATURE CITED}

Bassler, B. L., Wright, M., Showalter, R. E., and Silverman, M. R. 1993. Intercellular signalling in Vibrio harveyi: Sequence and function of genes regulating expression of luminescence. Mol. Microbiol. 9:773-86.

Bauer, W. D., and Robinson, J. B. 2002. Disruption of bacterial quorum sensing by other organisms. Curr. Opin. Biotechnol. 13:234-237.

Bauer, W. D., and Teplitski, M. 2001. Can plants manipulate bacterial quorum sensing? Aust. J. Plant Physiol. 28:913-921.

Cha, C., Gao, P., Chen, Y.-C., Shaw, P. D., and Farrand, S. K. 1998. Production of acyl-homoserine lactone quorum-sensing signals by gram- 
negative plant-associated bacteria. Mol. Plant Microbe Interact. 11:1119-1129.

Chen, X., Schauder, S., Potier, N., Van Dorsselaer, A., Pelczer, I., Bassler, B. L., and Hughson, F. M. 2002. Structural identification of a bacterial quorum-sensing signal containing boron. Nature 415:545-549.

Cook, D. R. 1999. Medicago truncatula-A model in the making! Curr. Opin. Plant Biol. 2:301-4.

Daniels, R., De Vos, D. E., Desair, J., Raedschelders, G., Luyten, E., Rosemeyer, V., Verreth, C. Schoeters, E., Vanderleyden, J., Michiels, J. 2002. The cin quorum sensing locus of Rhizobium etli CNPAF512 affects growth and symbiotic nitrogen fixation. J. Biol. Chem. 277:462-468.

Dworjanyn, S. A., de Nys, R., and Steinberg, P. D. 1999. Localization and surface quantification of secondary metabolites in the red alga Delisea pulchra. Marine Biol. 133:727-736.

Givskov, M., de Nys, R., Manefield, M., Gram, L., Maximilien, R., Eberl, L., Molin, S., Steinberg, P. D., and Kjelleberg, S. 1996. Eukaryotic interference with homoserine lactone-mediated prokaryotic signalling. J. Bacteriol. 178:6618-6622.

Joyce, E. A., Bassler, B. L., and Wright, A., 2000. Evidence for a signaling system in Helicobacter pylori: Detection of a luxS-encoded autoinducer. J. Bacteriol. 182:3638-43.

Kjelleberg, S., and Steinberg, P. 2002. Defenses against bacterial colonization of marine plants. In: Phyllosphere Microbiology. S. E. Lindow, E. Poinar, and V. Elliott, eds. American Phytopathological Society Press, St. Paul, MN, U.S.A.

Manefield, M., de Nys, R., Kumar, N., Read, R., Givskov, M., Steinberg, P., and Kjelleberg, S. 1999. Evidence that halogenated furanones from Delisea pulchra inhibit acylated homoserine lactone (AHL)-mediated gene expression by displacing the AHL signal from its receptor protein. Microbiol. 145:283-91.

Manefield, M., Harris, L., Rice, S. A., de Nys, R., and Kjelleberg, S. 2002. Halogenated furanones inhibit quorum sensing through accelerated LuxR turnover. Microbiol. 148:1119-27.

Marketon, M. M., Gronquist, M. R., Eberhard, A., and Gonzales, J. E., 2002. Characterization of the Sinorhizobium meliloti $\operatorname{sinR} / \sin I$ locus and the production of novel AHLs. J. Bacteriol. 184:5686-5695.

Mathesius, U., Mulders, S., Gao, M., Teplitski, M., Caetano-Anolles, G., Rolfe, B. G., and Bauer, W. D. 2003. Extensive and specific responses of a eukaryote to bacterial quorum sensing signals. Proc. Natl. Acad. Sci. U.S.A. 100:1444-1449.

McClean, K. H., Winson, M. K., Fish, L., Taylor, A., Chhabra, S. R., Camara, M., Daykin, M., Lamb, J. H., Swift, S., Bycroft, B. W., Stewart, G. S., and Williams, P. 1997. Quorum sensing and Chromobacterium violaceum: Exploitation of violacein production and inhibition for the detection of $N$-acylhomoserine lactones. Microbiol. 143:3703-11.

Miller, M. B., and Bassler, B. L., 2001. Quorum sensing in bacteria. Annu. Rev. Microbiol. 55:165-99.
Pierson, L. S., Wood, D. W., and von Bodman, S. B. 1999. Quorum sensing in plant-associated bacteria. Pages 101-115 in: Cell-Cell Signaling in Bacteria. G. M. Dunny and S. Winans, eds. American Society for Microbiology Press, Washington, D. C.

Rahme, L. G., Ausubel, F. M., Cao, H., Drenkard, E., Goumnerov, B. C., Lau, G. W., Mahajan-Miklos, S., Plotnikova, J., Tan, M. W., Tsongalis, J., Walendziewicz, C. L., and Tompkins, R. G. 2000. Plants and animals share functionally common bacterial virulence factors. Proc. Natl. Acad. Sci. U.S.A. 97:8815-21.

Schuster, M., Lostroh, C. P., Ogi, T., and Greenberg, E. P. 2003. Identification, timing, and signal specificity of Pseudomonas aeruginosa quorum-controlled genes: A transcriptome analysis. J. Bacteriol. 185:2066-2079

Steidle, A., Sigl, K., Schuhegger, R., Ihring, A., Schmid, M., Gantner, S., Stoffels, M., Riedel, K., Givskov, M., Hartmann, A., Langebartels, C., and Eberl, L. 2001. Visualization of $\mathrm{N}$-acylhomoserine lactone-mediated cell-cell communication between bacteria colonizing the tomato rhizosphere. Appl. Environ. Microbiol. 67:5761-70.

Surette, M. G., and Bassler, B. L. 1998. Quorum sensing in Escherichia coli and Salmonella typhimurium. Proc. Natl. Acad. Sci. U.S.A. 95:7046-50.

Teplitski, M., Robinson, J. B., and Bauer, W. D. 2000. Plants secrete substances that mimic bacterial $\mathrm{N}$-acyl homoserine lactone signal activities and affect population density-dependent behaviors in associated bacteria. Mol. Plant-Microbe Interact. 13:637-648.

Wagner, V. E., Bushnell, D., Passador, L., Brooks, A. I., and Iglewski, B. H. 2003. Microarray analysis of Pseudomonas aeruginosa quorumsensing regulons: Effects of growth phase and environment. J. Bacteriol. 185:2080-2095.

Whitehead, N. A., Barnard, A. M., Slater, H., Simpson, N. J. and Salmond, G. P., 2001. Quorum-sensing in gram-negative bacteria. FEMS (Fed. Eur. Microbiol. Soc.) Rev. 25:365-404.

Whiteley, M., Lee, K. M., and Greenberg, E. P., 1999. Identification of genes controlled by quorum sensing in Pseudomonas aeruginosa. Proc. Natl. Acad. Sci. U.S.A. 96:13904-9.

Winson, M. K., Swift, S., Fish, L., Throup, J. P., Jorgensen, F., Chhabra, S. R., Bycroft, B. W., Williams, P., and Stewart, G. S. 1998. Construction and analysis of luxCDABE-based plasmid sensors for investigating $N$-acyl homoserine lactone-mediated quorum sensing. FEMS (Fed. Eur. Microbiol. Soc.) Lett. 163:185-92.

Winzer, K., Hardie, K. R., and Williams, P. 2002. Bacterial cell-to-cell communication: Sorry, can't talk now-Gone to lunch! Curr. Opin. Microbiol. 5:216-22.

Zhu, J., and Winans, S. C. 2001. The quorum-sensing transcriptional regulator TraR requires its cognate signaling ligand for protein folding, protease resistance, and dimerization. Proc. Natl. Acad. Sci. U.S.A. 98:1507-12. 